Supplement of Adv. Stat. Clim. Meteorol. Oceanogr., 5, 57-66, 2019

https://doi.org/10.5194/ascmo-5-57-2019-supplement

(C) Author(s) 2019. This work is distributed under

the Creative Commons Attribution 4.0 License.

(c) (1)

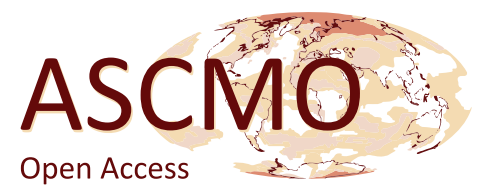

Supplement of

\title{
Fitting a stochastic fire spread model to data
}

\section{Joey Wang et al.}

Correspondence to: W. John Braun (john.braun@ubc.ca)

The copyright of individual parts of the supplement might differ from the CC BY 4.0 License. 


\section{Comparisons Between Simulated and Observed Data}
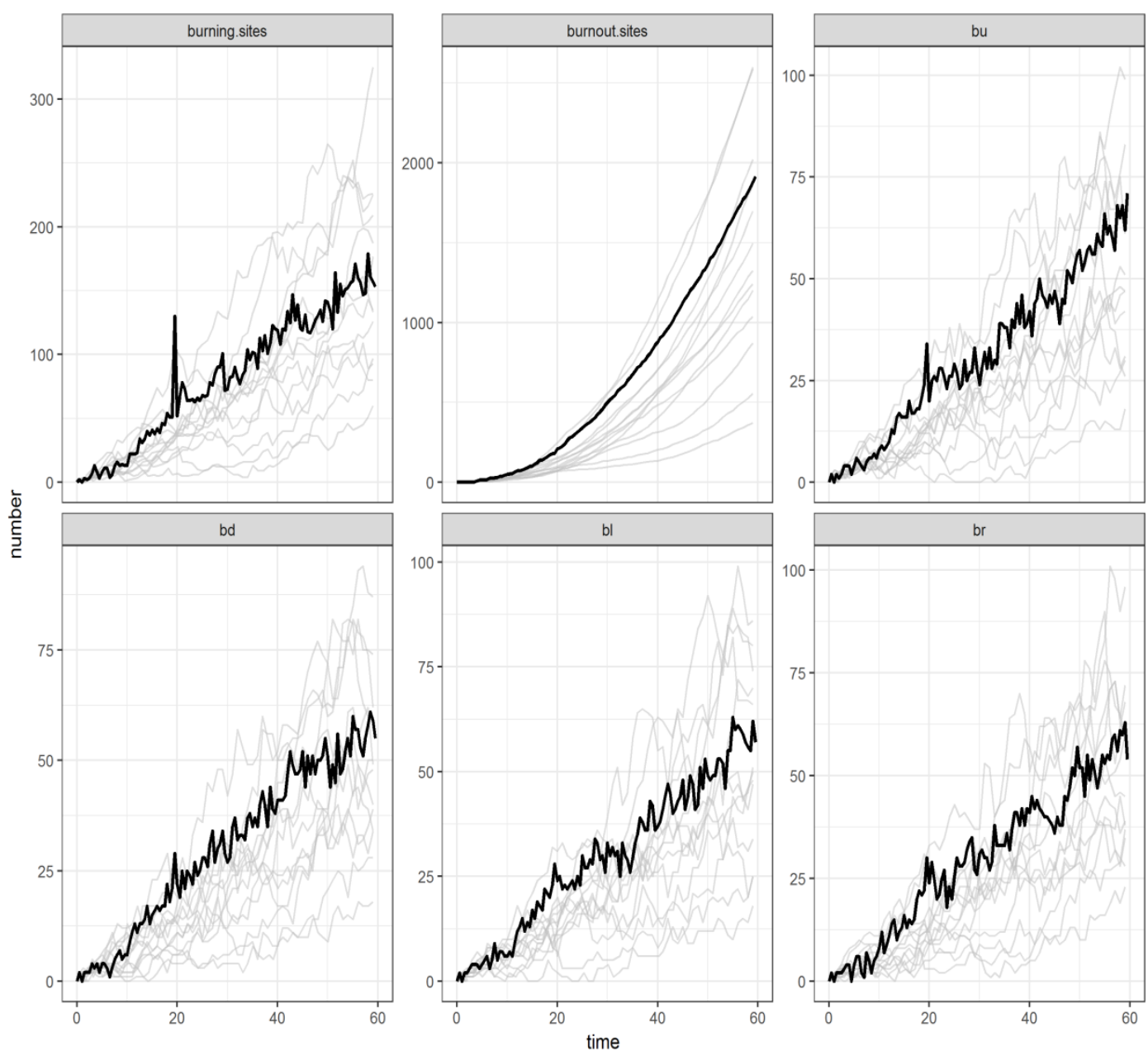

Supplementary Figure 1: Comparisons between data from 30 simulation runs and observed data from the first microfire. Top left: number of simulated burning sites (grey) versus time; middle top: Number of simulated burnt out sites (grey) versus time; remaining panels: numbers of simulated neighbourhood counts (grey) versus time. The black curve on each panel corresponds to the observed data from the first microfire, upon which the estimates of $\mu$ and $\lambda$ underlying the simulated data are based. 

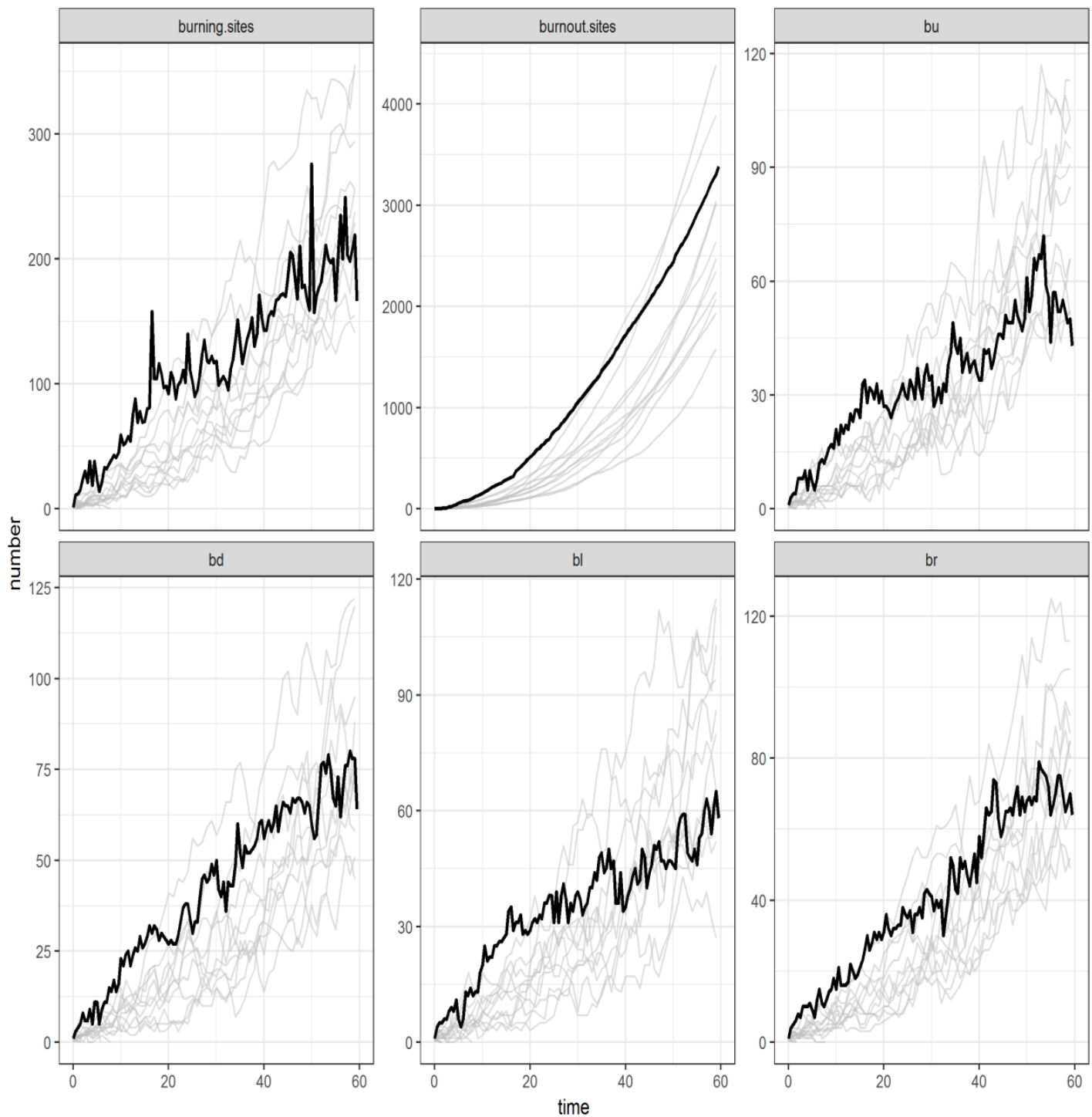

Supplementary Figure 2: Comparisons between data from 30 simulation runs and observed data from the second microfire. Top left: number of simulated burning sites (grey) versus time; middle top: Number of simulated burnt out sites (grey) versus time; remaining panels: numbers of simulated neighbourhood counts (grey) versus time. The black curve on each panel corresponds to the observed data from the first microfire, upon which the estimates of $\mu$ and $\lambda$ underlying the simulated data are based. 

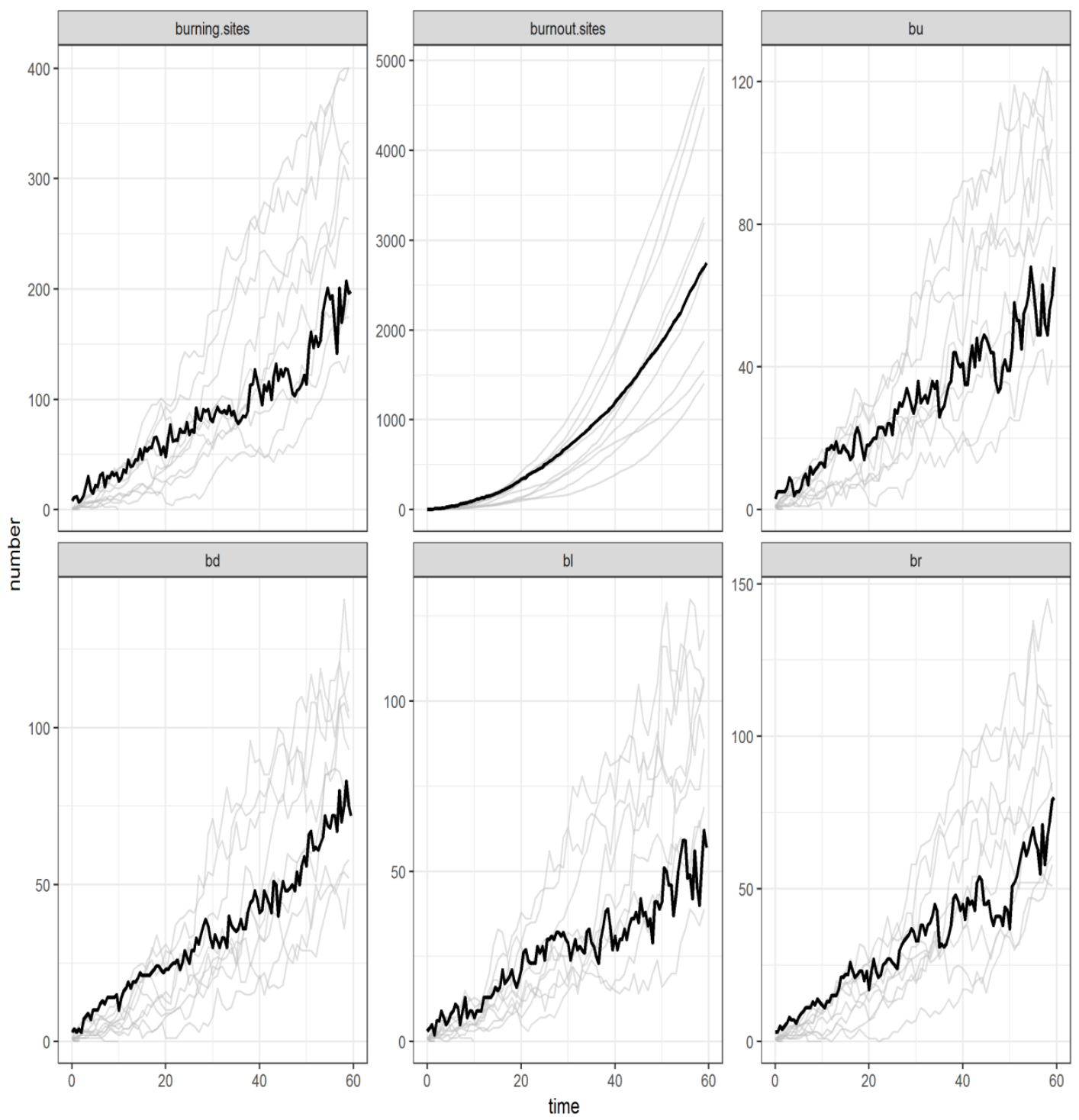

Supplementary Figure 3: Comparisons between data from 30 simulation runs and observed data from the third microfire. Top left: number of simulated burning sites (grey) versus time; middle top: Number of simulated burnt out sites (grey) versus time; remaining panels: numbers of simulated neighbourhood counts (grey) versus time. The black curve on each panel corresponds to the observed data from the first microfire, upon which the estimates of $\mu$ and $\lambda$ underlying the simulated data are based. 

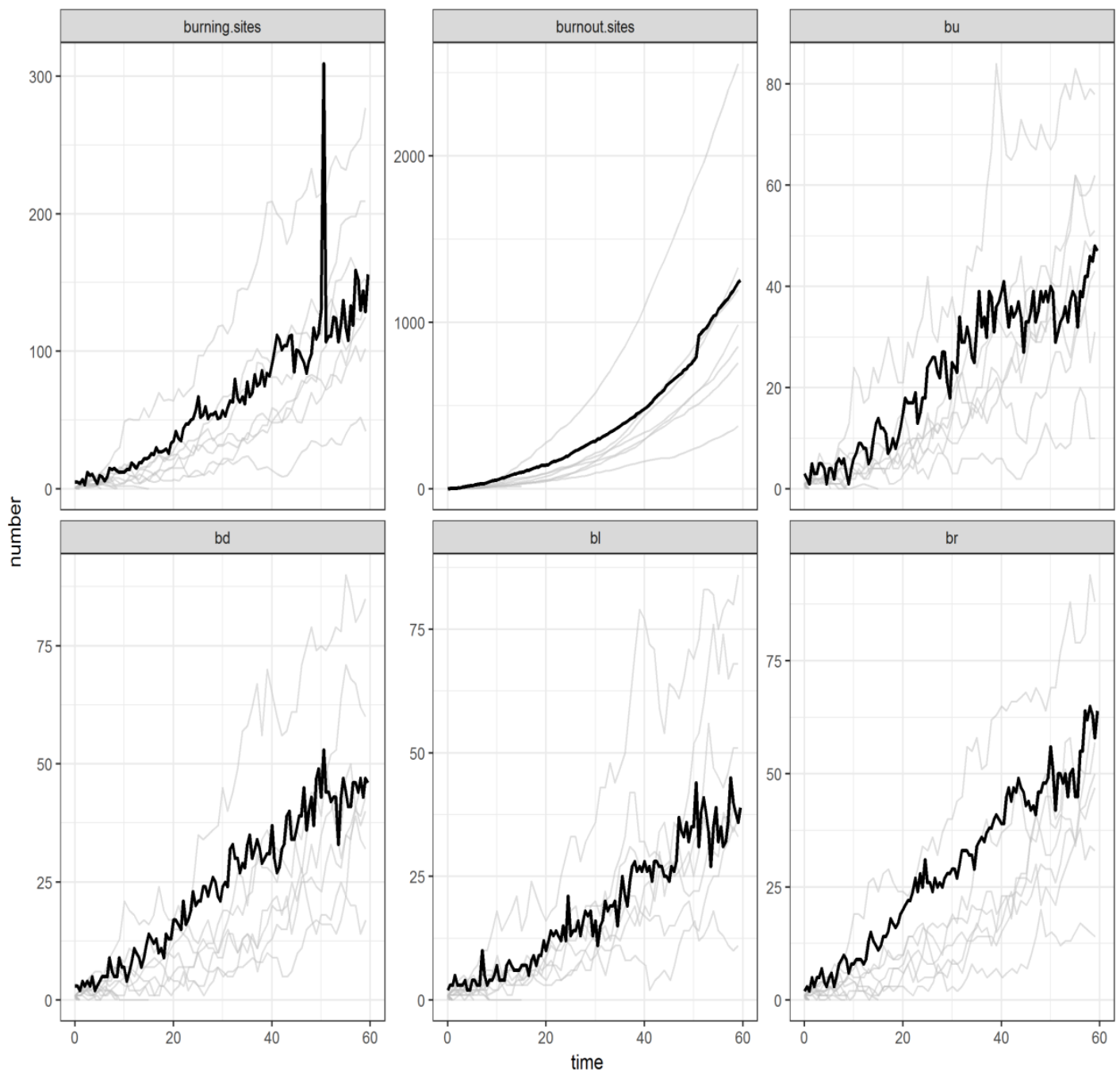

Supplementary Figure 4: Comparisons between data from 30 simulation runs and observed data from the fourth microfire. Top left: number of simulated burning sites (grey) versus time; middle top: Number of simulated burnt out sites (grey) versus time; remaining panels: numbers of simulated neighbourhood counts (grey) versus time. The black curve on each panel corresponds to the observed data from the first microfire, upon which the estimates of $\mu$ and $\lambda$ underlying the simulated data are based. 

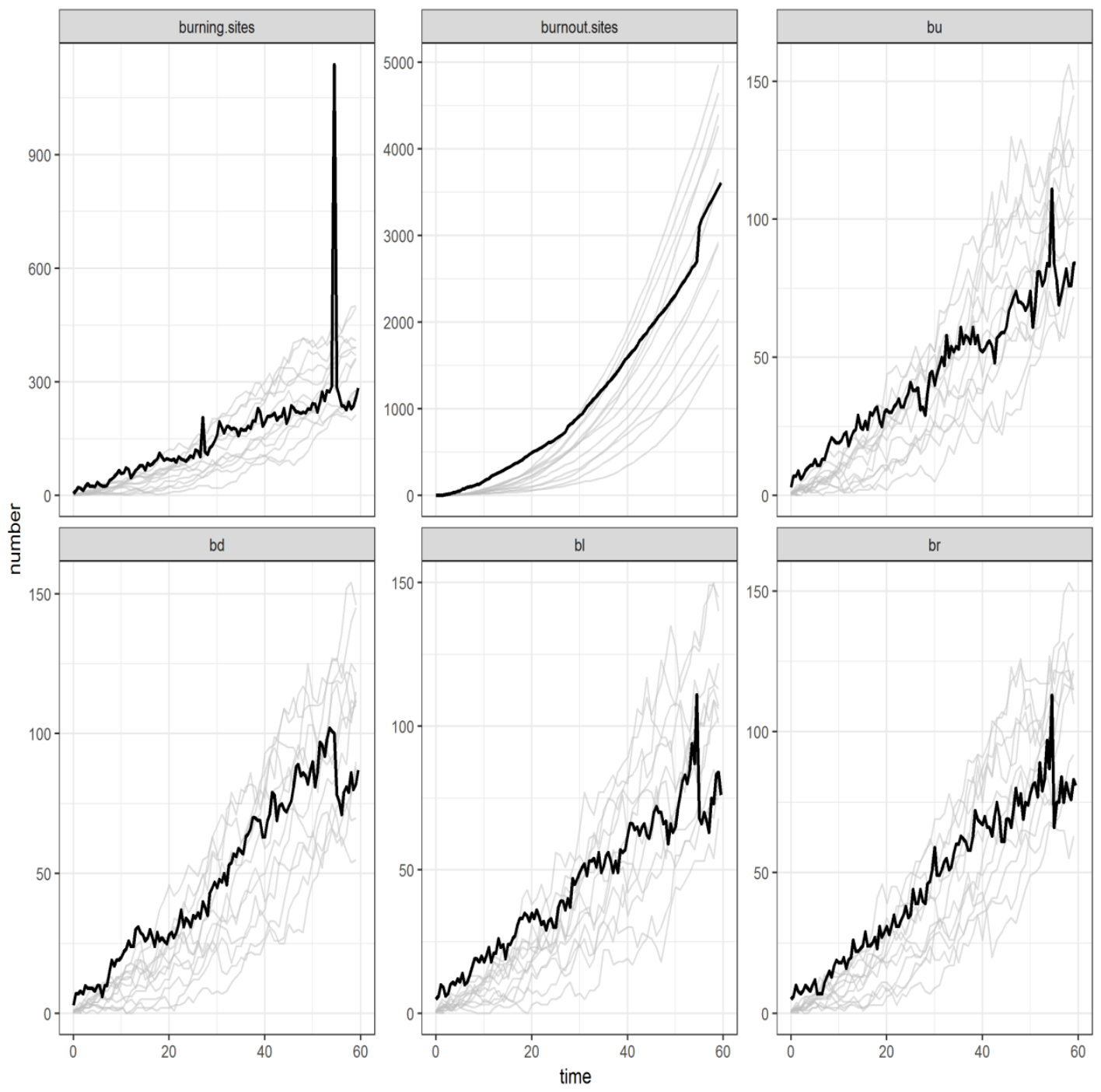

Supplementary Figure 5: Comparisons between data from 30 simulation runs and observed data from the fifth microfire. Top left: number of simulated burning sites (grey) versus time; middle top: Number of simulated burnt out sites (grey) versus time; remaining panels: numbers of simulated neighbourhood counts (grey) versus time. The black curve on each panel corresponds to the observed data from the first microfire, upon which the estimates of $\mu$ and $\lambda$ underlying the simulated data are based. 

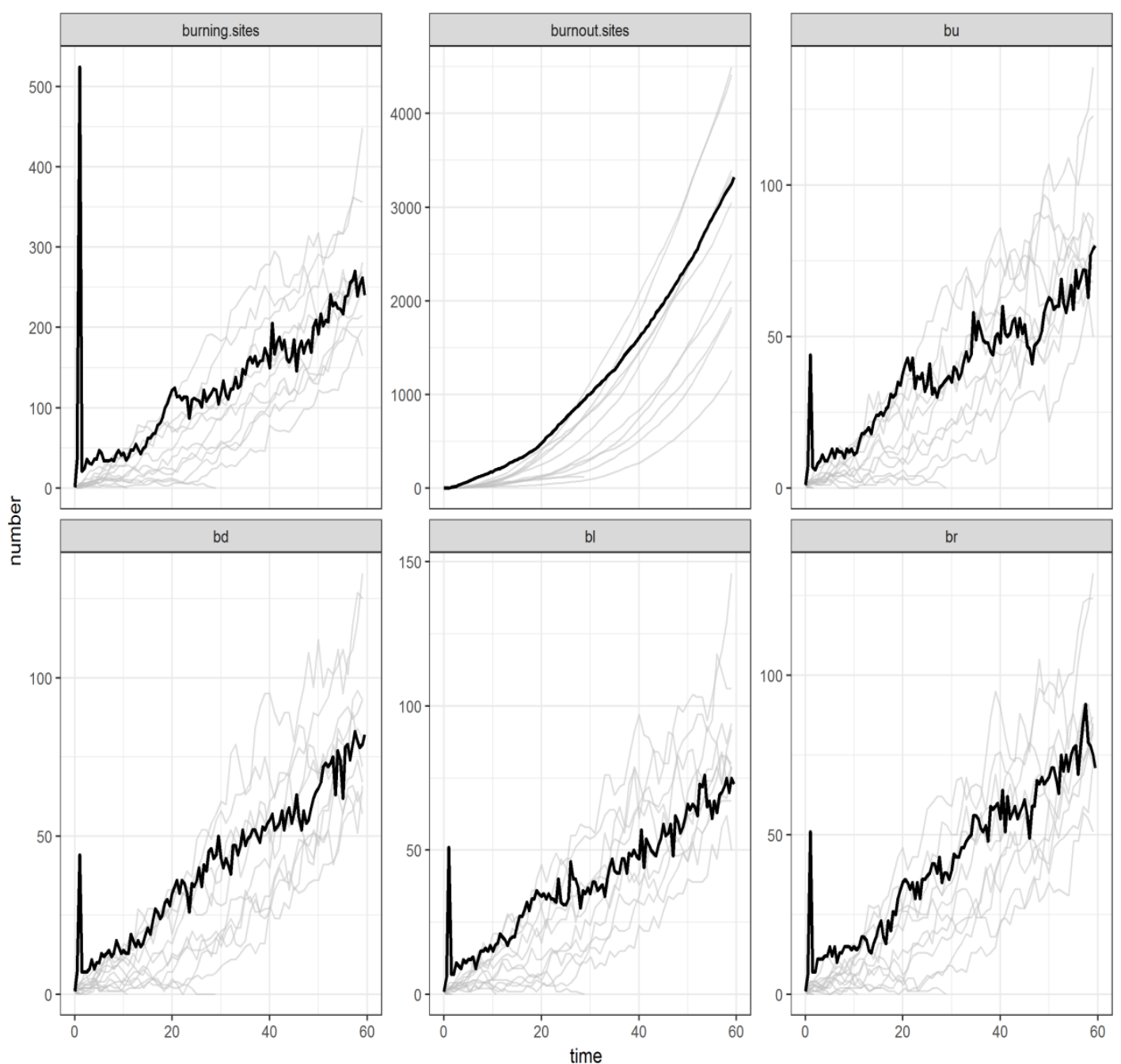

Supplementary Figure 6: Comparisons between data from 30 simulation runs and observed data from the sixth microfire. Top left: number of simulated burning sites (grey) versus time; middle top: Number of simulated burnt out sites (grey) versus time; remaining panels: numbers of simulated neighbourhood counts (grey) versus time. The black curve on each panel corresponds to the observed data from the first microfire, upon which the estimates of $\mu$ and $\lambda$ underlying the simulated data are based. 


\section{Further Assessment of Model Goodness of Fit}

According to the stochastic Boychuk model for fire spread, when a pixel is not burning, the time until it starts burning is exponentially distributed with parameter $k \lambda$ where $k$ is the number of neighbouring pixels that are burning $(k \in\{1,2,3,4\})$ and $\lambda$ is the exponential rate.

We cannot directly observe all of these waiting times, because they can be interrupted by the extinguishment of a neighbouring pixel before ignition of the pixel in question, or because a new neighbour is ignited leading to a higher risk of ignition at the site in question.

However, we can perform an approximate check of this exponential distribution assumption by examining each pixel and establishing the times at which $k$ of its 4 nearest-neighbours were ignited, for $k=1,2,3,4$. (The case where $k=4$ is not very interesting since it rarely occurs in the data; the fires we observe don't grow irregularly enough for this to happen very often, so we will omit this case).

We illustrate the concept with special cases for each value of $k=1,2,3$, as follows.

Suppose pixel $j$ has been ignited after one and only one of its 4 nearest neighbours, e.g. the one to the north, and the time of its ignition is $t_{j}$ and the times of ignition and extinguishment for the northern neighbour are $t_{N}$ and $T_{N}$ respectively.

The following quantity is either 0 or approximately exponentially distributed with parameter $\lambda$ according to the Boychuk model:

$$
t_{1}=\left(t_{j}-t_{N}\right) I_{t_{j}<T_{N}} .
$$

For the case where both the eastern and northern neighbours are burning, the following should be either 0 or approximately exponentially distributed with parameter $2 \lambda$ according to the Boychuk model: $\min \left\{t_{1}, t_{2}\right\}$ where

$$
\begin{aligned}
& t_{1}=\left(t_{j}-t_{N}\right) I_{t_{j}<T_{N}} . \\
& t_{2}=\left(t_{j}-t_{E}\right) I_{t_{j}<T_{E}} .
\end{aligned}
$$

For the case where both the southern, eastern and northern neighbours are burning, the following should be either 0 or approximately exponentially distributed with parameter $3 \lambda$ according to the Boychuk model: $\min \left\{t_{1}, t_{2}, t_{3}\right\}$ where

$$
\begin{aligned}
& t_{1}=\left(t_{j}-t_{N}\right) I_{t_{j}<T_{N}} . \\
& t_{2}=\left(t_{j}-t_{E}\right) I_{t_{j}<T_{E}} . \\
& t_{3}=\left(t_{j}-t_{S}\right) I_{t_{j}<T_{S}} .
\end{aligned}
$$

Exponential QQ-plots for these 3 types of quantities are supplied on the succeeding pages, for each of 6 microfires. The figures tell an interesting story. The $k=1 \mathrm{QQ}$-plots indicate that the quantity $t_{1}$, by itself, is not very well approximated by an exponential random variable, and it is not clear whether this is a result of censoring bias (see above) or a deficiency of the model. The $k=2$ and $k=3$ QQ-plots show that the corresponding quantities are well approximated by the corresponding exponential distributions. Thus, we have a mixed level of support for the Boychuk model, where the $k=1$ case may be indicating possible failure, and where the $k=2$ and $k=3$ cases may be indicating a surprising level of accuracy. 

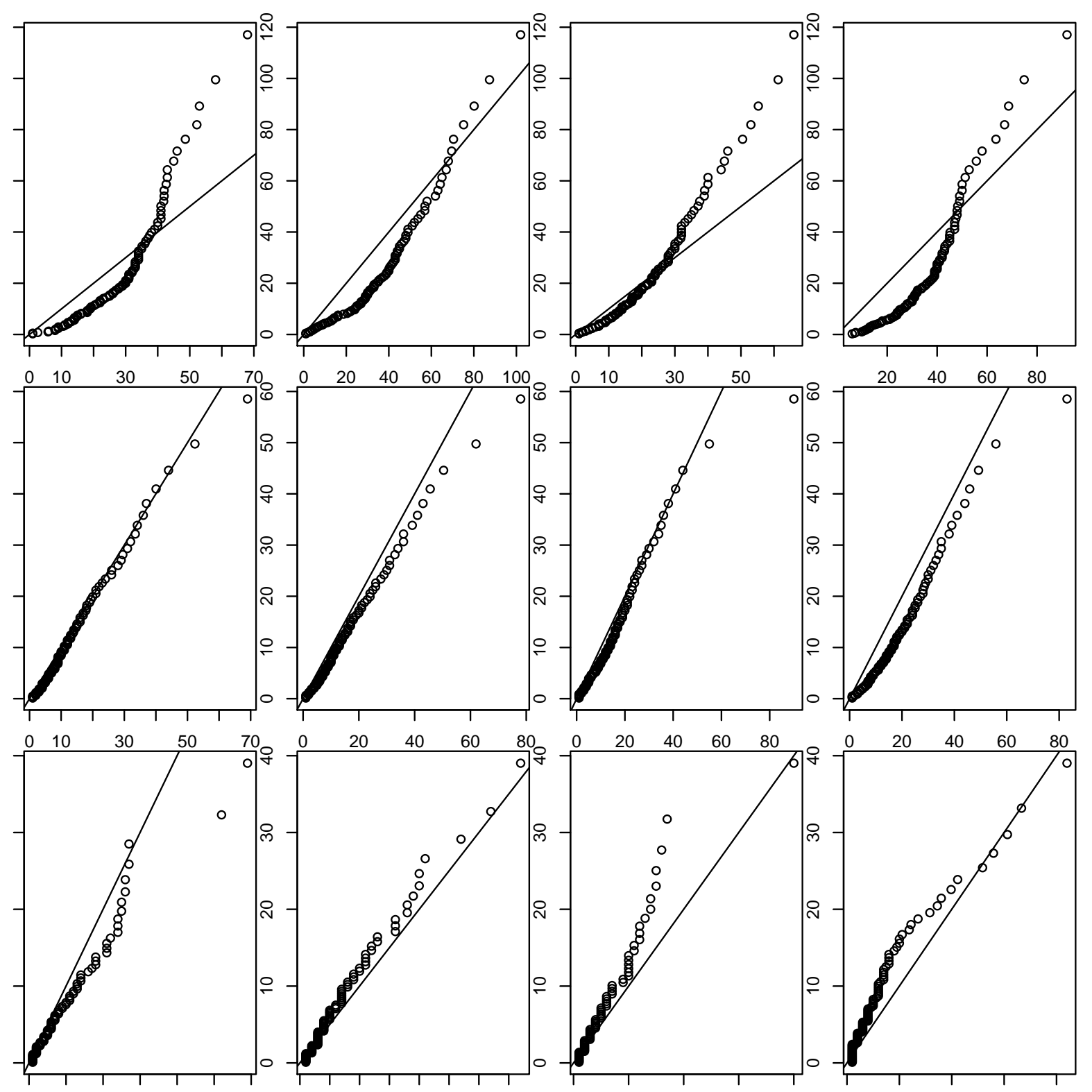

Supplementary Figure 7: Exponential QQ-plots for times to pixel ignition for the first microfire. First row of panels: QQ-plots for $t_{1}$ for each of the 4 compass directions, conditional on $t_{1}>0$. Reference line is based on the exponential distribution with parameter $\widehat{\lambda}$. Second row of panels: QQ-plots for the minimum of $t_{1}$ and $t_{2}$ for neighbouring compass directions, conditional on $t_{1}>0$ and $t_{2}>0$. Reference line is based on the exponential distribution with parameter $2 \widehat{\lambda}$. Third row of panels: QQ-plots for the minimum of $t_{1}, t_{2}$ and $t_{3}$, conditional on all three quantities being positive. Reference line is based on the exponential distribution with parameter $3 \hat{\lambda}$. 

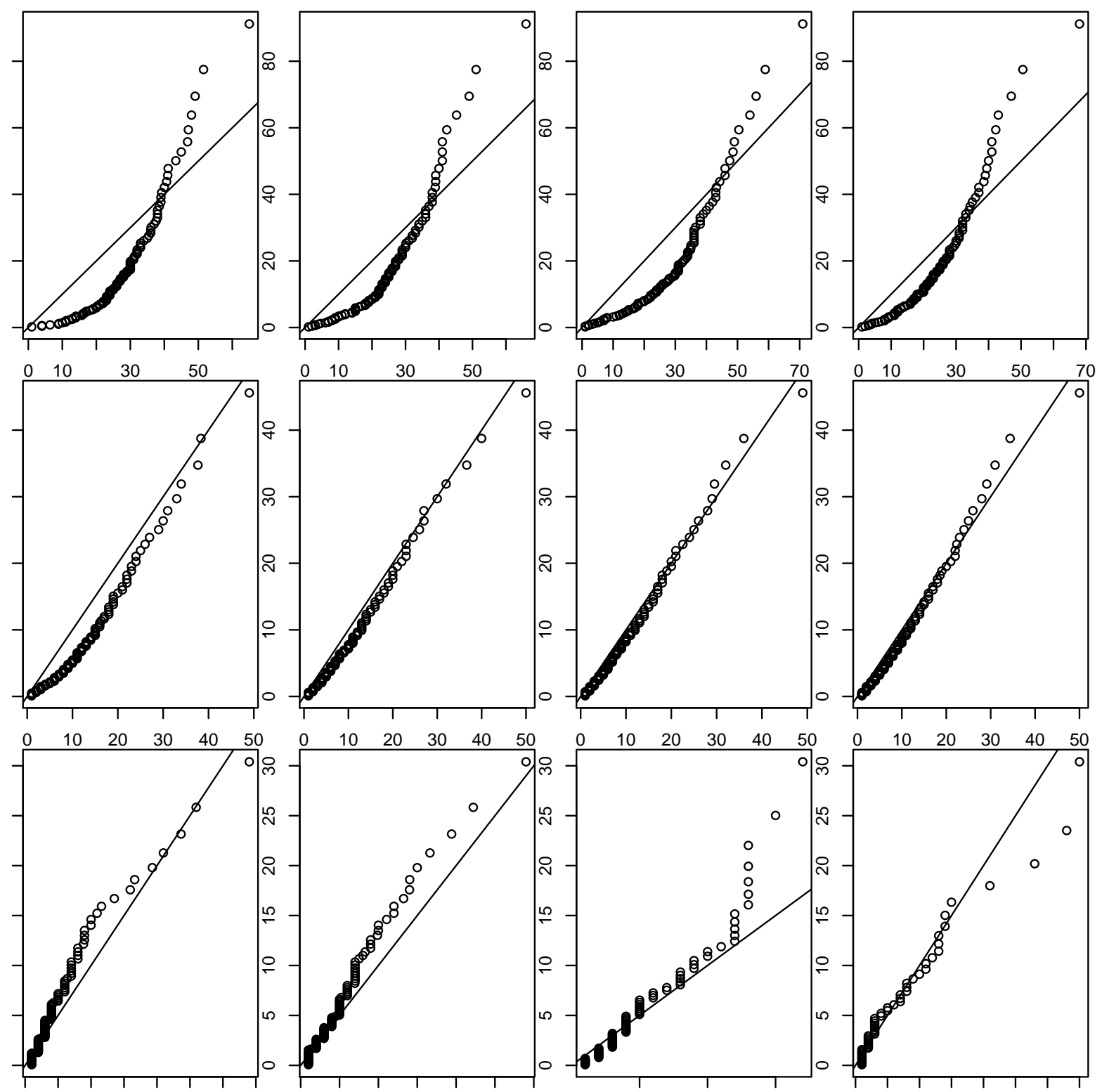

Supplementary Figure 8: Exponential QQ-plots for times to pixel ignition for the second microfire. First row of panels: QQ-plots for $t_{1}$ for each of the 4 compass directions, conditional on $t_{1}>0$. Reference line is based on the exponential distribution with parameter $\hat{\lambda}$. Second row of panels: QQ-plots for the minimum of $t_{1}$ and $t_{2}$ for neighbouring compass directions, conditional on $t_{1}>0$ and $t_{2}>0$. Reference line is based on the exponential distribution with parameter $2 \widehat{\lambda}$. Third row of panels: QQ-plots for the minimum of $t_{1}, t_{2}$ and $t_{3}$, conditional on all three quantities being positive. Reference line is based on the exponential distribution with parameter $3 \hat{\lambda}$. 


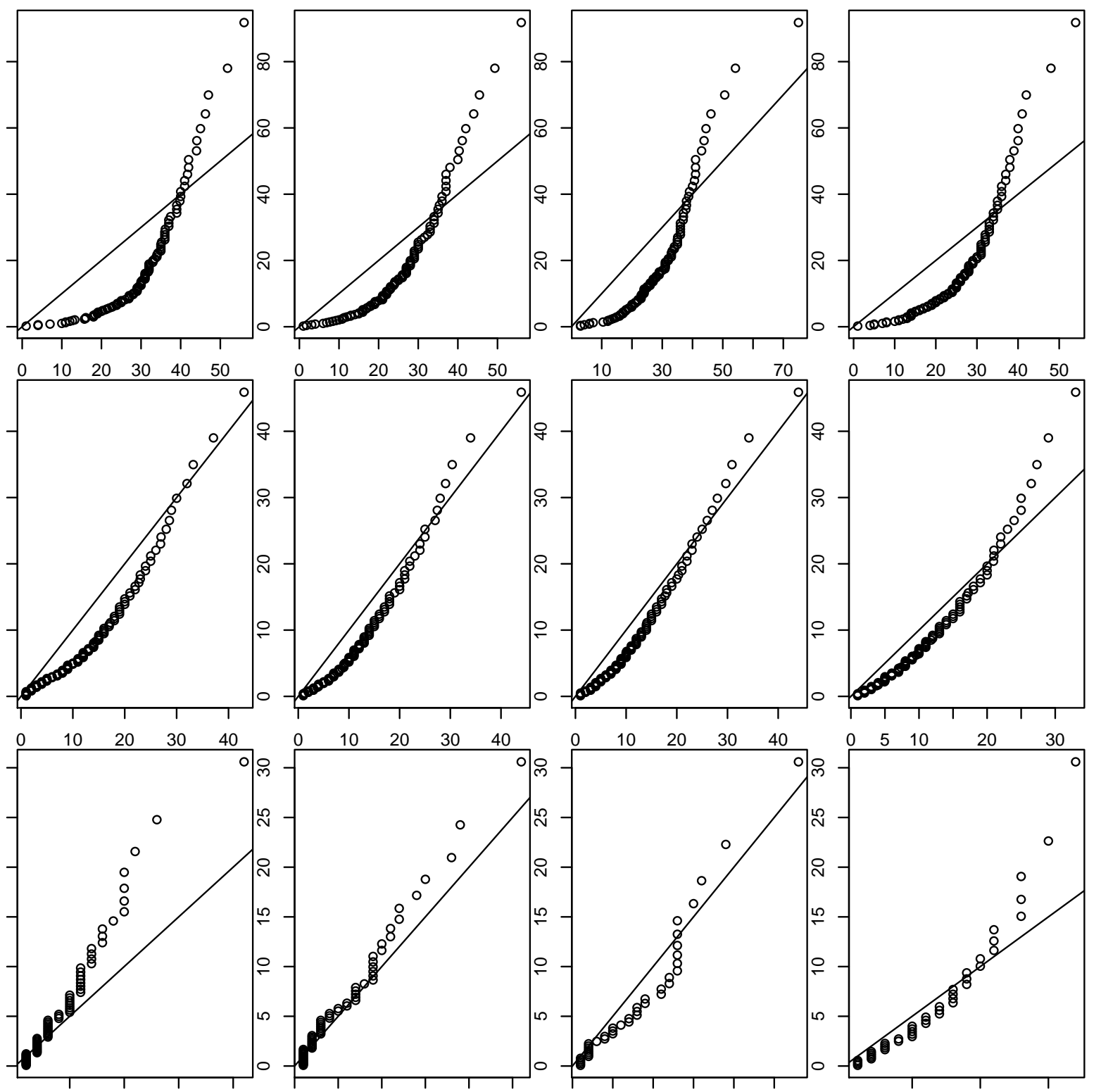

Supplementary Figure 9: Exponential QQ-plots for times to pixel ignition for the third microfire. First row of panels: QQ-plots for $t_{1}$ for each of the 4 compass directions, conditional on $t_{1}>0$. Reference line is based on the exponential distribution with parameter $\hat{\lambda}$. Second row of panels: QQ-plots for the minimum of $t_{1}$ and $t_{2}$ for neighbouring compass directions, conditional on $t_{1}>0$ and $t_{2}>0$. Reference line is based on the exponential distribution with parameter $2 \widehat{\lambda}$. Third row of panels: QQ-plots for the minimum of $t_{1}, t_{2}$ and $t_{3}$, conditional on all three quantities being positive. Reference line is based on the exponential distribution with parameter $3 \hat{\lambda}$. 

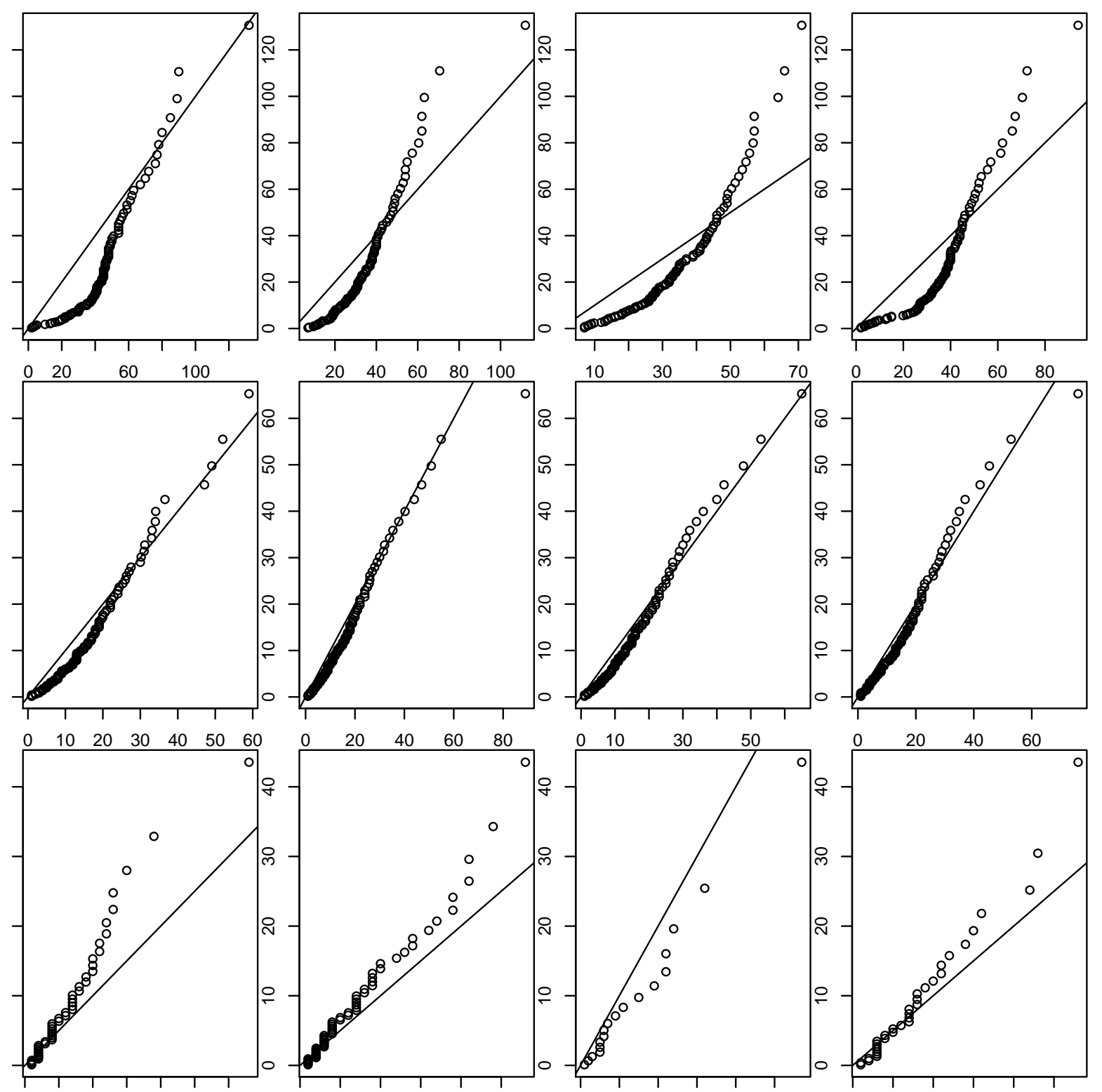

Supplementary Figure 10: Exponential QQ-plots for times to pixel ignition for the fourth microfire. First row of panels: QQ-plots for $t_{1}$ for each of the 4 compass directions, conditional on $t_{1}>0$. Reference line is based on the exponential distribution with parameter $\widehat{\lambda}$. Second row of panels: QQ-plots for the minimum of $t_{1}$ and $t_{2}$ for neighbouring compass directions, conditional on $t_{1}>0$ and $t_{2}>0$. Reference line is based on the exponential distribution with parameter $2 \widehat{\lambda}$. Third row of panels: QQ-plots for the minimum of $t_{1}, t_{2}$ and $t_{3}$, conditional on all three quantities being positive. Reference line is based on the exponential distribution with parameter $3 \hat{\lambda}$. 

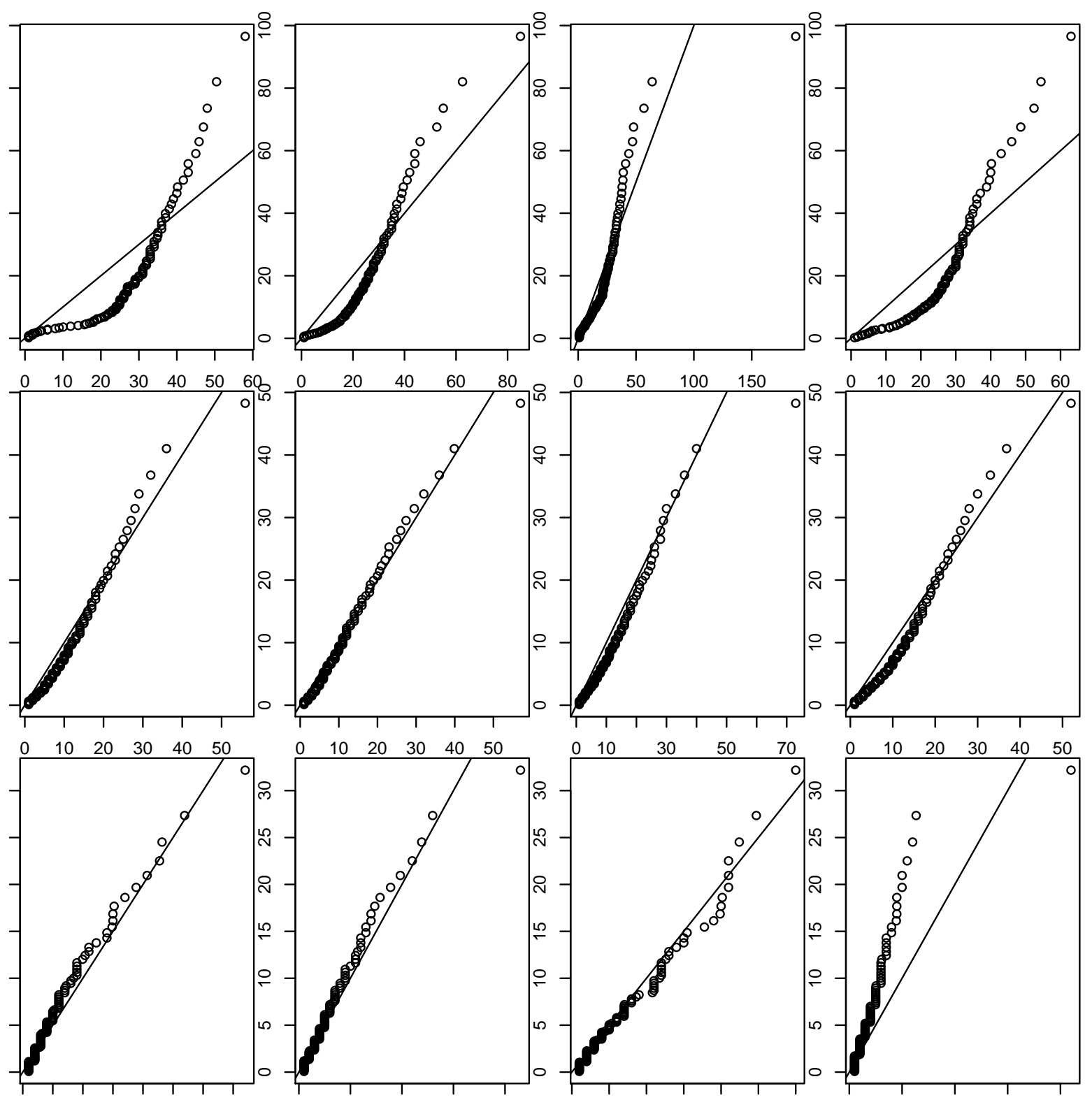

Supplementary Figure 11: Exponential QQ-plots for times to pixel ignition for the fifth microfire. First row of panels: QQ-plots for $t_{1}$ for each of the 4 compass directions, conditional on $t_{1}>0$. Reference line is based on the exponential distribution with parameter $\hat{\lambda}$. Second row of panels: QQ-plots for the minimum of $t_{1}$ and $t_{2}$ for neighbouring compass directions, conditional on $t_{1}>0$ and $t_{2}>0$. Reference line is based on the exponential distribution with parameter $2 \widehat{\lambda}$. Third row of panels: QQ-plots for the minimum of $t_{1}, t_{2}$ and $t_{3}$, conditional on all three quantities being positive. Reference line is based on the exponential distribution with parameter $3 \hat{\lambda}$. 

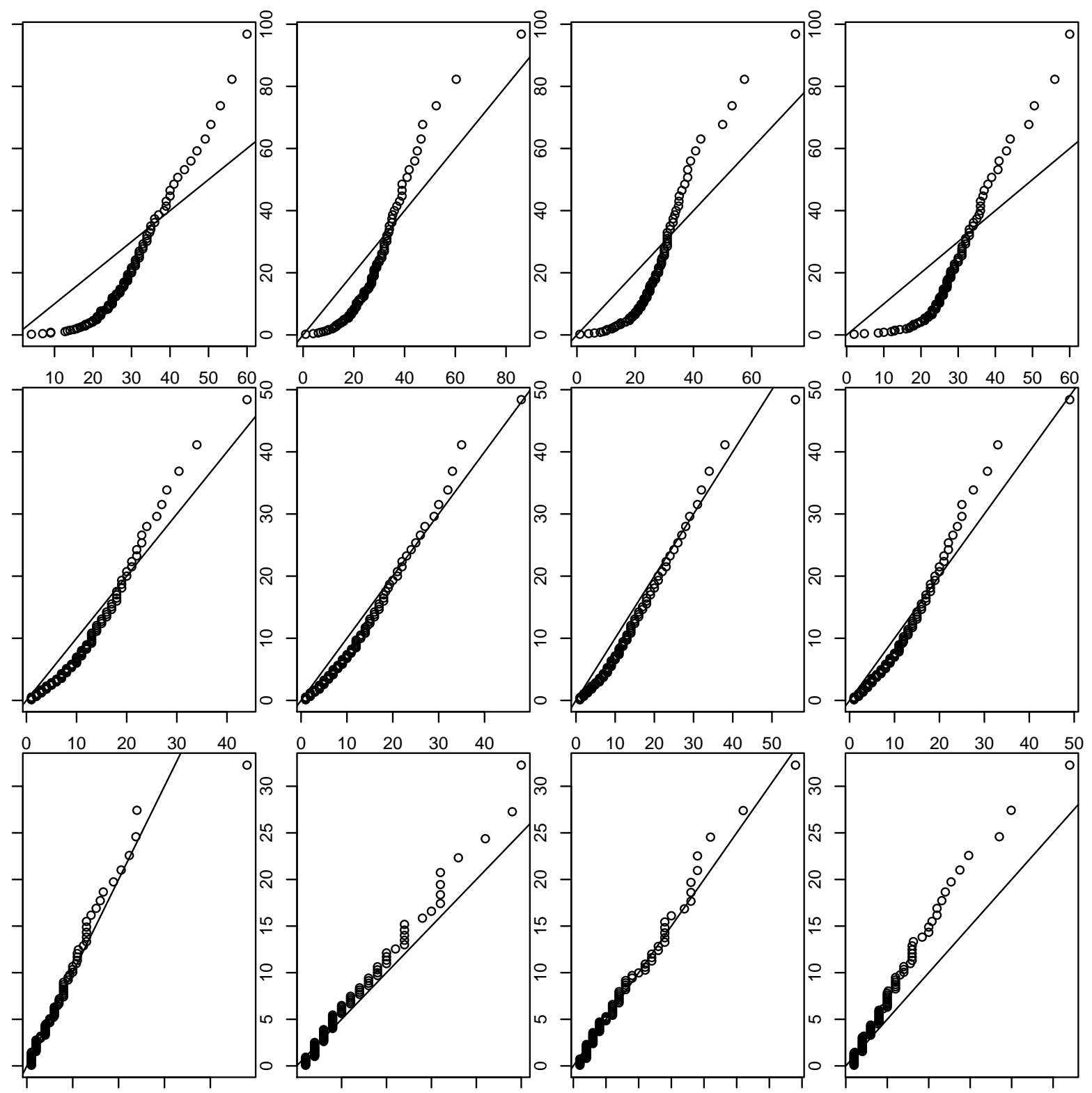

Supplementary Figure 12: Exponential QQ-plots for times to pixel ignition for the sixth microfire. First row of panels: QQ-plots for $t_{1}$ for each of the 4 compass directions, conditional on $t_{1}>0$. Reference line is based on the exponential distribution with parameter $\widehat{\lambda}$. Second row of panels: QQ-plots for the minimum of $t_{1}$ and $t_{2}$ for neighbouring compass directions, conditional on $t_{1}>0$ and $t_{2}>0$. Reference line is based on the exponential distribution with parameter $2 \widehat{\lambda}$. Third row of panels: QQ-plots for the minimum of $t_{1}, t_{2}$ and $t_{3}$, conditional on all three quantities being positive. Reference line is based on the exponential distribution with parameter $3 \hat{\lambda}$. 